\title{
Blind Digital Watermarking Framework Based on DTCWT and NSCT for Telemedicine Application
}

\author{
Nadhir Nouioua $^{1 *}$, Ali Seddiki ${ }^{1}$, Abdelkrim Ghaz $^{2}$ \\ ${ }^{1}$ Telecommunications and Digital Signal Processing Laboratory, Djillali Liabes University, Sidi Bel Abbes 22000, Algeria \\ ${ }^{2}$ Communications Networks Architecture and Multimedia Laboratory, Djillali Liabes University, Sidi Bel Abbes 22000, \\ Algeria
}

Corresponding Author Email: nadhir.nouioua@univ-sba.dz

https://doi.org/10.18280/ts.370608

Received: 19 October 2020

Accepted: 10 December 2020

\section{Keywords:}

blind watermarking, DTCWT, NSCT, quantization, robust, telemedicine

\begin{abstract}
In this work, a blind watermarking framework for medical images based on Dual-Tree Complex Wavelet Transform (DTCWT) and Non-subsampled Contourlet Transform (NSCT) is proposed. The core idea of this technique is to embed the watermark in the appropriate NSCT sub-band obtained by decomposing the cover image low coefficients purchased from the DTCWT standing on a quantization embedding function, the extraction phase is done without the requirement of the original cover image, what makes it a fully blind process. As clarity and integrity of the retrieved watermark are mandatory, a series of tests were exerted to affirm the robustness of the proposed scheme. The effectiveness of the watermarking is validated by using the peak signal-to-noise ratio (PSNR) and the structural similarity index measure (SSIM) through experiments. Simulation results demonstrate the invisibility of the proposed method and its strong robustness against various attacks, including additive noise, image filtering, JPEG compression, amplitude scaling, rotation attack, and combinational attack. Furthermore, the method in hands outperformance within the quantitative comparisons with other techniques in the literature in terms of rapid execution time, and quality extraction of hidden information, and appropriateness to be integrated for secure exchange in the healthcare sector.
\end{abstract}

\section{INTRODUCTION}

Since the dawn of history, humanity had lived many epidemics, wars and disasters, the recent pandemic lived by the mankind provoked by the Novel Coronavirus (COVID-19), had shown the importance of the Telemedicine, as this technology had provided huge facilities for human beings during confinement periods, in global such as medical prescriptions, medical advice, and others, one of the main branches of the Telemedicine is the Tele-radiology, where this tool has a powerful capability to help specialists in the medical field in pathologies interpretations and diagnosis. In the Telemedicine, a legitimate doctor's confidential reports and patients' information represented by Electronic Patient Records (EPR), should be carefully protected and kept far away from any kind of tampering. Medical images like any other type of data need a certain amount of security and protection against maleficent use, what makes experts face a vivid ethical responsibility to protect this information from stealing, looting, and operating in any manner outside the owner's authority.

In fact, most digital images dispatched via networks are not immune from copying and misusing. Researchers have worked in this concept by inspiring a method from Steganography which is considered as an art of data hiding [1], this inspired method is called watermarking [2]. Watermarking is the process of embedding a certain data into host support [3], this technique may function in both domains frequency and spatial [4], where each domain provide specific advantages such as robustness against ambiguity attacks [5], geometrical attacks [6] and compression [7], and another factor which is the imperceptibility, where the watermarked image should be subjectively clear for the user [8]. Miscellaneous watermarking schemes exist, those schemes can be segmented into two groups Fragile watermarking and Robust watermarking, the first one operates directly on image pixels, where it can lead to high imperceptibility and less robustness, it is generally based on Least Significant Bit (LSB) $[9,10]$, for the second group the watermark is inserted in the frequency coefficients gained by the transformations, it reports high robustness facing signal processing distortions and high levels of imperceptibility $[11,12]$. Furthermore, the two classes may be hybridized such as in the study [13].

Recently, researchers suggested multifold digital watermarking techniques, in next, some frameworks are discussed. Ernawan and Kabir [14], suggested a scheme based on Discrete Cosine Transform (DCT) psycho-visual threshold, the method is about picking some frequency areas from the DCT for the embedding procedure in reason of avoiding image high deformations, where the selected frequencies choice was based on visual and edge entropy, also Arnold chaotic map is exerted on the watermark for security reinforcement. This technique shows good robustness against several attacks except for rotation, Poonam and Arora [15] proposed a watermarking technique established on Discrete Wavelet Transform (DWT), and Singular Value Decomposition (SVD), the watermark was incorporated within singular values gained from the DWT decomposition. The indicated method revealed 
significant robustness above signal processing attacks. Islam et al. [16], embedded the watermark in a third level Lifting Wavelet Transform (LWT), selected sub-band, and this last, was blended then divided into quadrants and randomized, where different secret keys are used for both operations, in the other part a Support Vector Machine (SVM) was utilized for watermark detection. Here, results are sufficient in terms of robustness and imperceptibility. A Convolutional Neural Network (CNN) based watermarking algorithm designed by Li et al. [17], the watermark is firstly embedded in the DCT component then for the extraction stage a Cooperative Neural Network was applied. Optimal imperceptibility was guaranteed by this method. Abraham and Paul [18], proposed a method in the spatial domain for colored images, the watermark was divided into sub-blocs and even the host image Blue channel was divided into sub-images, where each watermark sub-bloc was embedded in a sub-image, this method stands on two masks, one for the embedding and the other for compensation. Results achieved by this algorithm shows poor resilience versus cropping.

The literature presents, a Dual-Tree Complex Wavelet Transform and Non-subsampled Contourlet Transform based for blind digital watermarking, the procedure is about decomposing the host image utilizing the DTCWT, then exerting the NSCT on the Low frequencies sub-band gained from the DTCWT, from the fruitful of the NSCT, a suitable sub-band is selected to embed a binary watermark, where the used color space is YIQ that is used in the National Television Standard Committee (NTSC), and the Chrominance Component (Q) is the chosen component for the watermark embedding. With no prior knowledge about the hidden-mark, applying the same decompositions as the embedding process will lead the receiver to the watermark. A deeper understanding of the Embedding/ Extraction procedures is drawn in section 3 .

The major impact of this paper is a blind reversible watermarking scheme that provides elevated imperceptibility levels which is an essential pillar in medical images due to its sensitivity, and also the robustness against ambiguity attacks and compression. Intensive series of attacks were conducted on the proposed method, for performances evaluation the used metrics are Peak Signal to Noise Ratio, Mean Square Error, Structural Similarity Index Image (SSIM), Normalized Correlation (NC), and Bit Error Rate (BER), as proof of the robustness of the method in hands a comparison with recent works is also done.

The rest of this paper is organized as follows. Background of the used materials in section 2. The proposed scheme is elucidated intensely in section 3 . In section 4 , the performance results and quantitative comparisons are discussed and illustrated. The work is concluded in section 5 .

\section{BASIC KNOWLEDGE}

In this section, an overview of the DTCWT and NSCT is illustrated in detail for each tool, the choice of the materials was based on the furnished characteristics defined in the sequel.

\subsection{Dual tree complex wavelet transform}

Kingsbury [19], came out with the Dual-Tree Complex Wavelet Transform which is the DWT complex development [20]. The DTCWT fuse the DWT and CWT (Complex
Wavelet Transform) criterions [21], where the main delivered advantages are translation invariance and favorable directional selectivity [22]. Figure 1 shows the DTCWT structure and coefficients orientations.

Mathematically speaking, the DTCWT apply two parallel DWTs simultaneously to obtain the real and imaginary parts one from each branch respectively [23]. Shift insensibility assured by the DTCWT, can yield to a watermarking scheme resilient facing geometric attacks, moreover, the appraising operation of real and imaginary parts done by the DTCWT, and these will point the way to phase information. The main difference between the real and imaginary parts is demonstrated in the filter banks construction, all way by taking in consideration that if the branches uses the same filters nothing will be achieved, moreover, filter banks are very complex to be designed, and also more memory is needed while computation. The DTCWT function is defined as:

$$
\begin{gathered}
\varphi(x, y)=\varphi_{o}(x) \varphi_{o}(y)-\varphi_{b}(x) \varphi_{b}(y)+ \\
J\left[\varphi_{o}(x) \varphi_{b}(y)+\varphi_{b}(x) \varphi_{o}(y)\right] \\
\operatorname{Real}\{\varphi(x, y)\}=\varphi_{o}(x) \varphi_{o}(y)-\varphi_{b}(x) \varphi_{b}(y) \\
\text { Imagingary }\{\varphi(x, y)\}=\varphi_{o}(x) \varphi_{b}(y)-\varphi_{b}(x) \varphi_{o}(y)
\end{gathered}
$$

Orthogonal and biorthogonal real wavelets are denoted $\varphi_{o}$ and $\varphi_{b}$ consecutively.

Long story short, the DTCWT principle is about exerting filters on image rows and columns to engender low-pass subbands (approximation) and high-pass sub-bands (details) [24], where the diverse details sub-bands directionalities provided by the DTCWT are $\pm 15^{\circ}, \pm 45^{\circ}$ and $\pm 75^{\circ}$ [25]. The directionality feature insured by the DTCWT, make image unresponsive to coefficients variations that provokes artifacts, and which will lead to high fidelity retrieved watermarks.

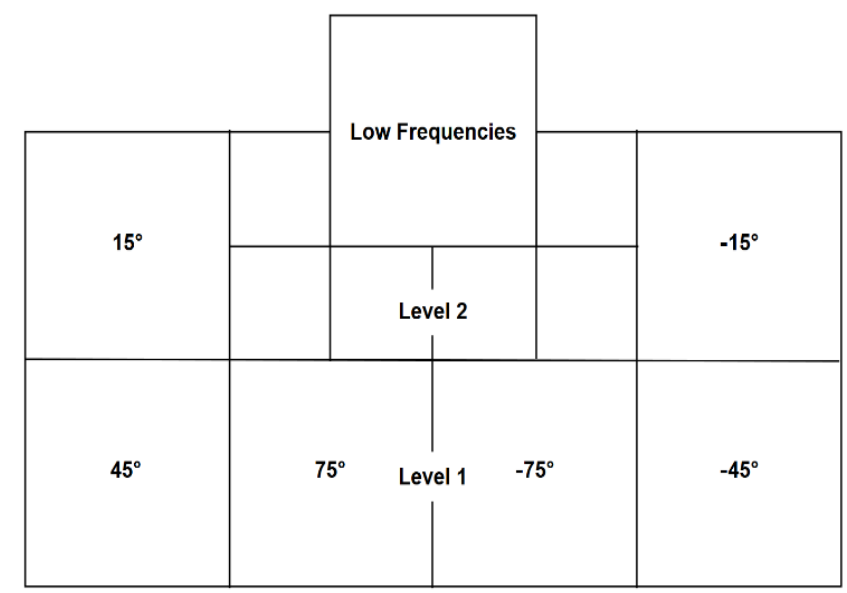

Figure 1. DTCWT level 2 design

\subsection{Non-subsampled contourlet transform}

The Contourlet Transform (CT) was first presented by Do and Vetteli [26], which is a multi-resolution and multidirectional transform, contours are better exhibited by the Contourlet Transform analogized to divers transforms [27]. Da Cunha et al. [28] suggested NSCT, which is the shift invariant model of the CT [29], besides that, in the course of 
image decomposing and reconstructing, the NSCT abolish down and up samples [30], the Figure 2 shows the NSCT structure.

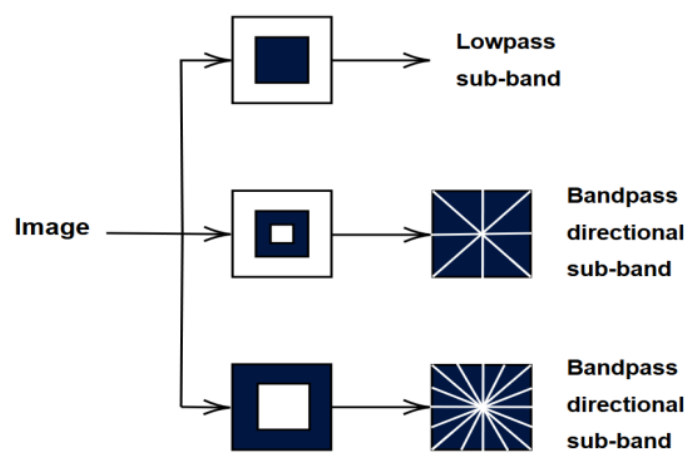

Figure 2. Non-subsampled contourlet transform skeleton

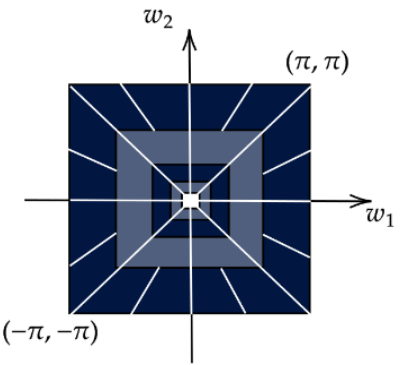

Figure 3. 2D illustration of frequency segregation utilizing NSDFB

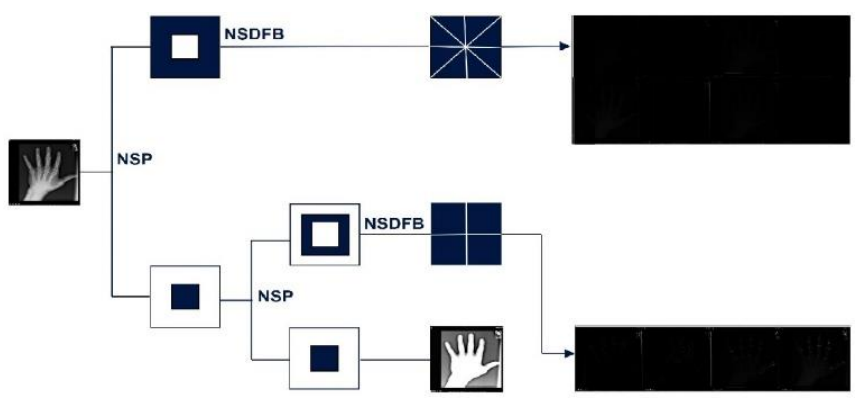

Figure 4. Demonstration of the NSCT workflow

With the aim of reaching multi-scale decomposition, the Non-subsampled Pyramid (NSP), is applied on the image, the result of this operation is low frequencies and high frequencies, then to obtain multi-directional decomposition (see Figure 3), Non-subsampled Directional Filter Bank (NSDFB) is exerted on the high-frequencies for each scale [30]. Low-pass coefficients is denoted $K^{L P}$, and directional high-pass coefficient $K_{s, d}^{H P}$, where s represents scale and d the direction index. Figure 4, explains the NSCT workflow graphically.

\section{PROPOSED WATERMARKING FRAMEWORK}

Within the tremendous progress in the computer security realm. This paper proposed a blind digital watermarking algorithm to ensure authentication and copyright protection. As briefly presented in the introduction, this method is based on the DTCWT-NSCT in YIQ color space. The advantages behind combining those transforms are excellent directional selectivity and translation invariant. There are divers watermarking solutions employing either DTCWT or NSCT using different embedding rules, poor results were extrapolated from exploiting those transforms alone, which leads to the conception of cascading the transforms in hands (DTCWT and NSCT), proposed Embedding and extraction procedures are displayed in Figure 5. Mechanisms details are described in the following sub-sections.

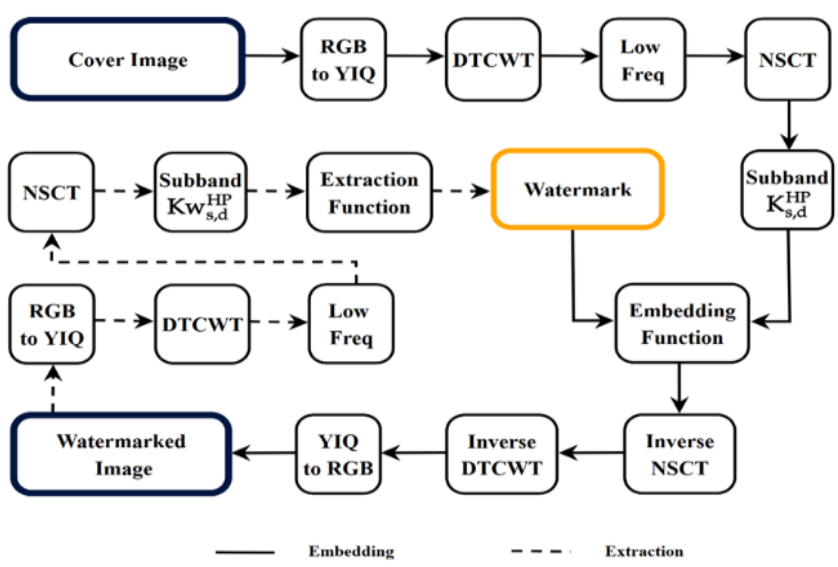

Figure 5. Embedding and extraction processes flowchart of the proposed scheme

\subsection{Watermark embedding mechanism}

In the embedding scheme, the binary watermark is incorporated in a color cover image. At first, the cover image is converted into YIQ color space, next the DTCWT is applied on the (Q) component, the low frequencies sub-band is decomposed using NSCT, where a high frequencies sub-band is selected for the watermark embedding using a quantization embedding function, in order to obtain the watermarked image, the inverse of the transforms was exerted. The exact embedding steps are given below:

1. Read the cover image and the watermark

2. Convert the cover image from the RGB color space into YIQ color space using the next formula:

$$
\left[\begin{array}{l}
Y \\
I \\
Q
\end{array}\right]=\left[\begin{array}{ccc}
0.299 & 0.587 & 0.114 \\
0.596 & -0.274 & -0.322 \\
0.211 & -0.523 & 0.312
\end{array}\right]\left[\begin{array}{l}
R \\
G \\
B
\end{array}\right]
$$

3. Exert the DTCWT second level on the chrominance component $(\mathrm{Q})$

4. Apply the NSCT on the low pass sub-band obtained from step 3, using three levels $(0,2,3)$, and the used filters are "pkva"

5. Quantify selected high-pass sub-band tagged $K_{1,3}^{H P}$ purchased from the NSCT, as follows:

$$
Q(i, j)=\bmod \left(\left(\operatorname{round}\left(\frac{K_{1,3}^{H P}}{\nabla}\right)\right), 2\right)
$$

6. Embed the watermark denoted $\mathrm{W}$ using the next formulas:

$$
C(i, j)=K_{1,3}^{H P} \quad \text { if } \quad Q(i, j)=W(i, j)
$$




$$
C(i, j)=\left\{\begin{array}{ccc}
K_{1,3}^{H P}-\nabla & \text { if } & K_{1,3}^{H P} \geq 0 \\
K_{1,3}^{H P}+\nabla & \text { else } & K_{1,3}^{H P}<0
\end{array}\right.
$$

$\mathrm{C}$ is the embedding matrix, $\mathrm{i}$ and $\mathrm{j}$ represents coordinates.

The factor $\nabla$ should be carefully selected since a big quantization step will make the algorithm more robust and less imperceptible and vice versa.

The function round included in MATLAB libraries' spins results into its nearest figures.

7. Apply inverse of each transform (NSCT, DTCWT and YIQ) to reconstruct the watermarked image.

\subsection{Watermark extraction mechanism}

For the purpose of going backward and retrieving the watermark, the extraction process requires only the received watermarked image, the essential steps for extraction are summarized as follows:

1. Read the watermarked image

2. Convert the watermarked image from the RGB color space into YIQ color space

3. Decompose chrominance component (Q) using DTCWT level 2
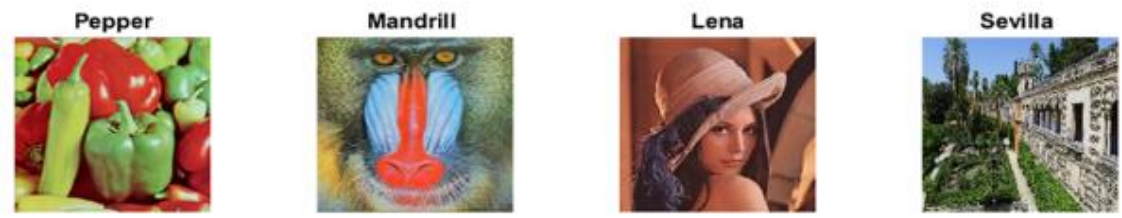

Hand

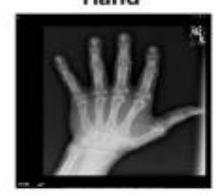

Thorax 2

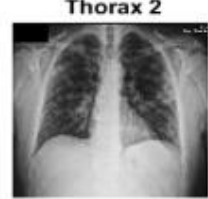

Heart

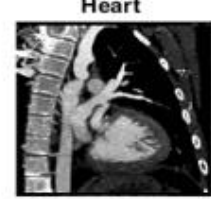

Thorax 3

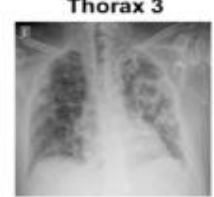

4. Perform NSCT on the result of step 3 low frequencies sub-band

5. Extract the watermark from the sub-band $K w_{1,3}^{H P}$ using the next based quantization equation:

$$
W_{\text {extracted }}=\left\{\begin{array}{c}
1 \text { if } \bmod \left(\left(\operatorname{round}\left(\frac{K w_{1,3}^{H P}(i, j)}{\nabla}\right)\right), 2\right)=1 \\
0 \text { elsewhere }
\end{array}\right.
$$

\section{EXPERIMENTAL RESULTS}

The simulation was executed with MATLAB R2019a as a software, utilizing Intel ${ }^{\circledR}$ i5-4200U 1.6GHz CPU, and 6Go of RAM as hardware, a set of medical images collected from the study [31], and non-medical images, shown in Figure 6, are used as host images, the used watermarks are shown in Figure 7. The host image and the watermark sizes are $512 \times 512$ and $32 \times 32$ respectively. The evaluation metrics used are described in the coming sub-section, the proposed method was subjected to various attack types with different parameters, method robustness was compared with other works.

Figure 6. Medical and non-medical test images

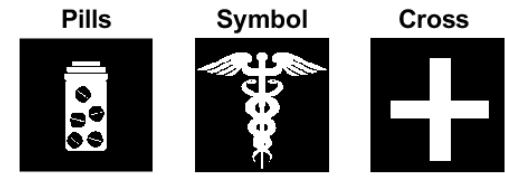

Figure 7. Watermarks set

\subsection{Evaluation metrics}

For the sake of measuring high fidelity and robustness, some powerful metrics in the image processing field were employed, in reason of making a fair judgment on the proposed work.

- Mean Square Error (MSE): Determines mean error magnitude between two images, it can be calculated using the next equation:

$$
M S E=\frac{1}{M \times N} \sum_{0}^{M-1} \sum_{0}^{N-1}(C(i, j)-W(i, j))^{2}
$$

$\mathrm{M}$ and $\mathrm{N}$ are image dimensions, and $\mathrm{C}$ and $\mathrm{W}$ represent the cover image and the stego-image respectively

- Peak signal to Noise Ratio (PSNR): Calculates the error among the original cover image and the watermarked image, mathematically is given by:

$$
P S N R=10 \log _{10}\left(\frac{P e a k^{2}}{M S E}\right)
$$

where, Peak is the maximum value within the data. 
- Normalized Correlation (NC): Often used to demonstrate similarity amongst the embedded and retrieved watermark

$$
N C=\frac{\sum_{i=1}^{x} \sum_{j=1}^{y}\left(W_{o r}(i, j) \times W_{e x}(i, j)\right)}{\sum_{i=1}^{x} \sum_{j=1}^{y} W_{o r}^{2}(i, j)}
$$

where, $W_{\text {or }}$ represent the embedded watermark and $W_{\text {ex }}$ the extracted one

- Structural Similarity Index (SSIM): is a perceptual metric that allows to compute difference between watermarked image and a reference image, which is given by:

$$
\operatorname{SSIM}(I, \hat{I})=\frac{\left(2 \mu_{I} \mu_{\hat{I}}+c_{1}\right)\left(2 \sigma_{\hat{I}}+c_{2}\right)}{\left(\mu_{I}^{2}+\mu_{\hat{I}}^{2}+c_{1}\right)\left(\sigma_{I}^{2}+\sigma_{\widehat{I}}^{2}+c_{2}\right)}
$$

where, $I$ represent the reference image, $\hat{I}$ signify the watermarked image. The average is represented by $\mu$, the variance of the original image and the watermarked image is denoted as $\sigma_{I}$ and $\sigma_{\hat{I}}$, and $\sigma_{I \hat{I}}$ is the covariance, while $c_{I}$ and $c_{2}$ are stabilization variables.

- $\quad$ Bite Error Rate (BER): Described as the number of errors divided by the entirety watermark bits, its defined by:

$$
B E R=\frac{\text { Errors }}{\text { Numberof bits }}
$$

\subsection{Imperceptibility analysis}

The bar graph in Figure 8, and Table 1 show the PSNR results under different quantization coefficients' (0.1-0.5), the experiments were conducted to choose the most convenient quantization step $\nabla$. The used coefficient within this method was not based only on the high imperceptibility but also the robustness delivered, the choice fell on $\nabla=0.4$, which assures satisfactory results in terms of imperceptibility, and robustness as demonstrated downstream, some watermarked image and their extracted watermarks samples utilizing the elected $\nabla$ factor are illustrated in Figure 9. Table 2 shows the results of the proposed method in terms of imperceptibility and robustness, the proposed work provides an average PSNR of 35.80 , the results were revealed using quantization coefficient $\nabla=0.4$, as stated earlier, this coefficient provides good image quality and high robustness.

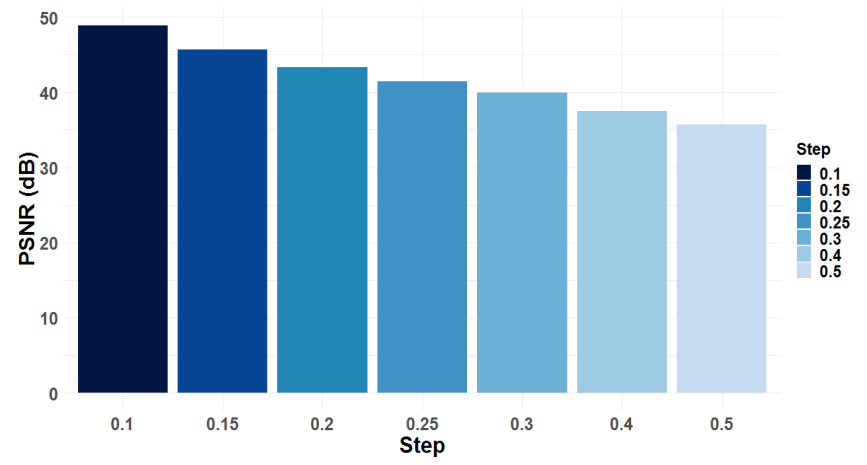

Figure 8. PSNR results under varying quantization coefficients

Table 1. PSNR $(\mathrm{db})$ of some image samples using different quantization steps

\begin{tabular}{cccccccc}
\hline Step $(\boldsymbol{\nabla})$ & Pepper & Mandrill & Lena & Lowlight & Skull & Thorax1 & Head \\
\hline 0.1 & 46.109 & 49.562 & 50.123 & 49.792 & 49.863 & 51.350 & 45.622 \\
0.15 & 43.369 & 46.290 & 46.914 & 46.695 & 46.530 & 47.828 & 42.110 \\
0.2 & 41.338 & 44.044 & 44.578 & 44.316 & 44.115 & 45.329 & 39.617 \\
0.25 & 39.785 & 42.176 & 42.630 & 42.345 & 42.184 & 43.391 & 37.683 \\
0.3 & 38.680 & 40.680 & 41.074 & 40.722 & 40.608 & 41.807 & 36.103 \\
0.35 & 36.764 & 37.828 & 38.581 & 37.466 & 38.138 & 39.308 & 34.299 \\
0.4 & 35.174 & 36.346 & 36.651 & 36.379 & 36.197 & 37.370 & 31.675 \\
\hline
\end{tabular}

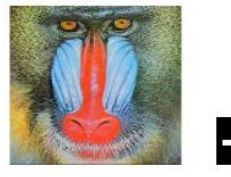

$\boldsymbol{\Psi}$
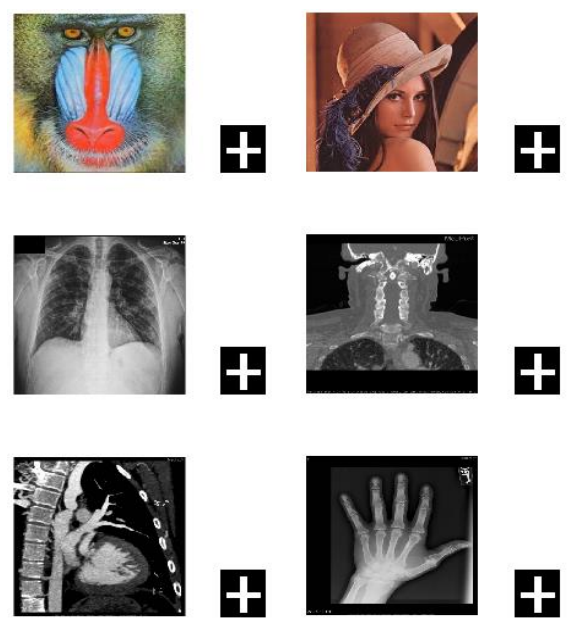

Figure 9. Watermarked image and extracted watermark using quantization $\nabla=0.4$ step
Table 2. No-attack proposed scheme results

\begin{tabular}{cccccc}
\hline Metric & $\begin{array}{c}\text { PSNR } \\
\text { (dB) }\end{array}$ & MSE & SSIM & NC & BER \\
\hline Pepper & 36.764 & 0.000211 & 1 & 0.99 & 0 \\
Mandrill & 37.828 & 0.000165 & 1 & 1 & 0 \\
Lena & 38.581 & 0.000139 & 1 & 1 & 0 \\
Sevilla & 37.613 & 0.000173 & 1 & 1 & 0 \\
Lowlight & 37.466 & 0.000179 & 1 & 1 & 0 \\
Hand & 33.984 & 0.0004 & 1 & 1 & 0 \\
Heart & 34.013 & 0.000397 & 1 & 0.99 & 0 \\
Stomach & 33.722 & 0.000424 & 1 & 1 & 0 \\
Skull & 38.138 & 0.000154 & 1 & 1 & 0 \\
Thorax 1 & 39.308 & 0.000117 & 1 & 1 & 0 \\
Thorax 2 & 33.765 & 0.000420 & 1 & 0.99 & 0 \\
Thorax 3 & 33.721 & 0.000424 & 1 & 1 & 0 \\
Neck & 33.985 & 0.000399 & 1 & 1 & 0 \\
Head & 34.299 & 0.000372 & 1 & 0.98 & 0 \\
Brain & 33.901 & 0.000407 & 1 & 1 & 0 \\
\hline
\end{tabular}




\subsection{Robustness analysis}

In this part robustness of the proposed method is analyzed by applying several attacks utilizing the $\mathrm{NC}$ and BER as objective metrics and also subjectively by the visual quality of the retrieved watermarks. Table 4 reveals results of 15 various attacks (see Figure 10 and Table 3 to remark the differences between the original and the attacked images), the suggested scheme delivers high figures in terms of normalized correlation and low BER, as well as the watermark elevated quality. Correspondingly, and in reason of testing the present mechanism's effectiveness, the scheme was examined under different signal processing manipulations using variable noise densities and JPEG compression quality factors. Figure 11 illustrates the proposed method resistance facing \& Pepper noise at different densities, the NC value of \& Pepper noise with $10 \%$ density is 0.82 , which is high enough to recognize the extracted watermark.

As shown in Figure 12, the proposed algorithm has certain immunity against Gaussian noise densities starting from 0.001 till 0.02, then NC values start decreasing with significant BER increasing. This method depicted dazzling robustness versus Speckle noise under different densities, as stated in Figure 13. Furthermore, for JPEG compression (see Figure 14), the BERs values slightly increased at quality factors 30 and 40, with NC values superior or equal to 0.8 , BERs values are less than 0.03 . The experimental results of the proposed method under different attacks using variable noise density affirms its robustness.

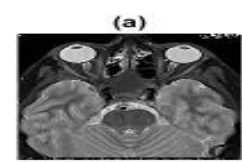

(d)

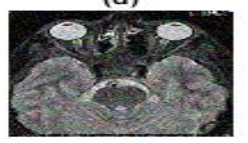

(g)

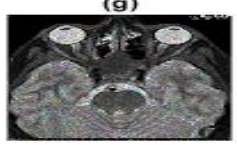

(j)

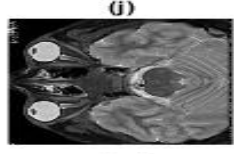

(m)
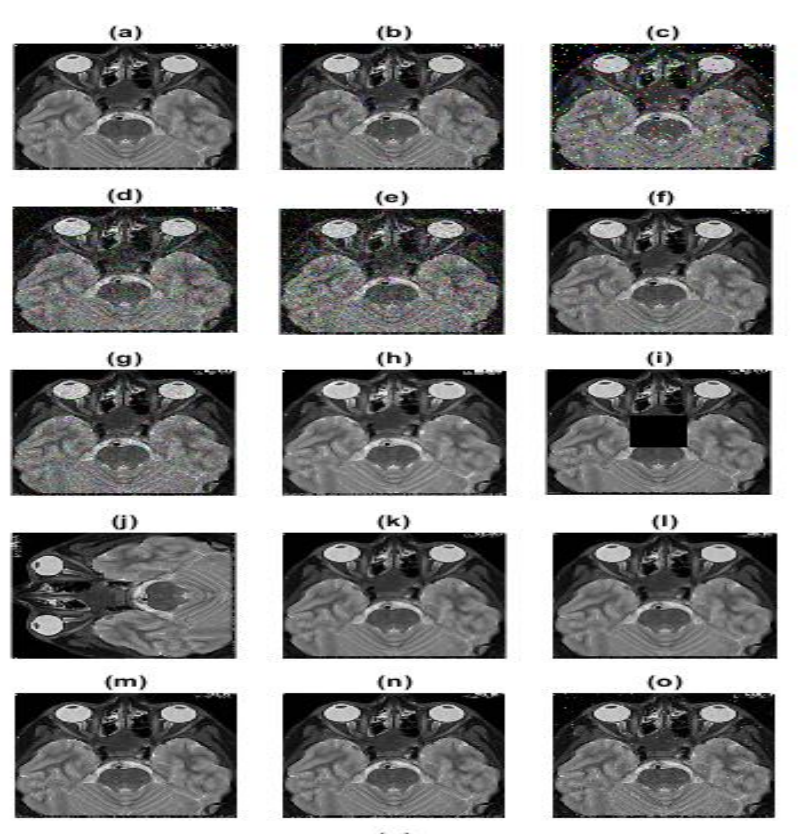

(f)

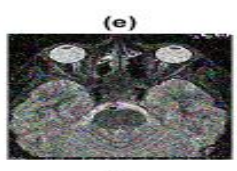

(h)

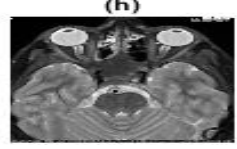

(k)
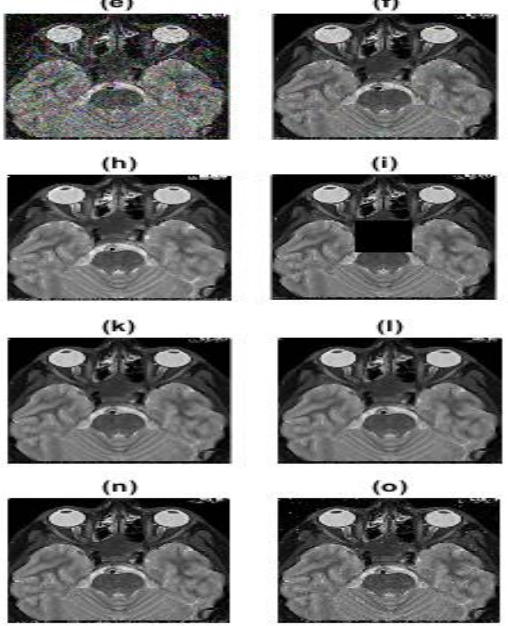

(p)

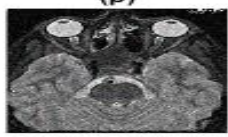

Figure 10. (a) Original watermarked image, (b) \& pepper (density $=1 \%$, (c) \& pepper (density=5\%), (d) Gaussian noise (mean $=0$; density=1\%), (e) Gaussian noise (mean=0; density=2\%), (f) Speckle noise (density=1\%), (g) Speckle noise $($ variance $=5 \%)$, (h) Dilatation $(5,5)$, (i) Cropping (1/16), (j) Rotation $\left(90^{\circ}\right)$, (k) Median Filtering (5,5), (1)

Median Filtering (3,3), (m) JPEG Compression (40), (n) JPEG Compression (80), (o) JPEG Compression (80) and \& Pepper (1\%), (p) JPEG Compression (80) and Gaussian noise $(1 \%)$
Table 3. Illustration of PSNR and MSE results for watermarked image in reference with the original host image

\begin{tabular}{|c|c|c|}
\hline Attacks & $\begin{array}{l}\text { PSNR } \\
(\text { dB })\end{array}$ & MSE \\
\hline Salt \& Pepper $($ density $=1 \%)$ & 24.150 & 0.0038 \\
\hline Salt \& Pepper $($ density $=5 \%)$ & 17.715 & 0.0169 \\
\hline Gaussian noise $($ mean $=0 ;$ density $=1 \%)$ & 20.331 & 0.0093 \\
\hline Gaussian noise $($ mean $=0$; density $=2 \%)$ & 17.577 & 0.0175 \\
\hline Speckle noise $($ density $=1 \%)$ & 27.236 & 0.0019 \\
\hline Speckle noise $($ variance $=5 \%)$ & 21.214 & 0.0076 \\
\hline Dilatation $(\mathbf{5 , 5})$ & 22.913 & 0.0051 \\
\hline Cropping (1/16) & 20.093 & 0.0098 \\
\hline Rotation (90॰) & 11.241 & 0.0751 \\
\hline Median Filtering $(\mathbf{5 , 5})$ & 28.932 & 0.0013 \\
\hline Median Filtering $(3,3)$ & 31.048 & $\begin{array}{c}0.0000 \\
7\end{array}$ \\
\hline JPEG Compression (40) & 33.033 & $\begin{array}{c}0.0000 \\
4\end{array}$ \\
\hline JPEG Compression (80) & 34.757 & $\begin{array}{c}0.0000 \\
3\end{array}$ \\
\hline $\begin{array}{c}\text { JPEG Compression (80) and Salt \& } \\
\text { Pepper }(1 \%)\end{array}$ & 26.956 & 0.0020 \\
\hline $\begin{array}{c}\text { JPEG Compression (80) and Gaussian } \\
\text { noise }(1 \%)\end{array}$ & 22.779 & 0.0053 \\
\hline
\end{tabular}

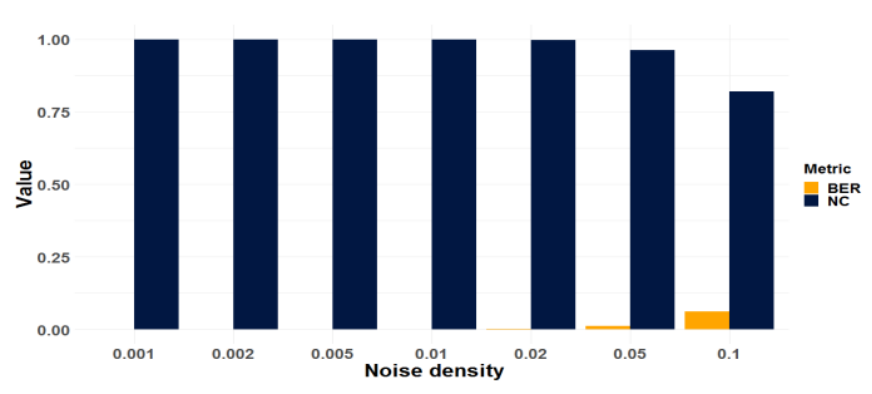

Figure 11. Lena image robustness results against Salt \& Pepper noise different densities

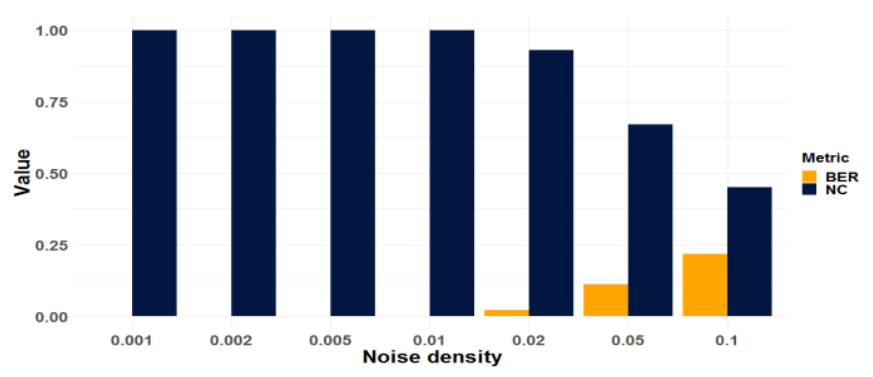

Figure 12. Lena image robustness results against Speckle noise different densities

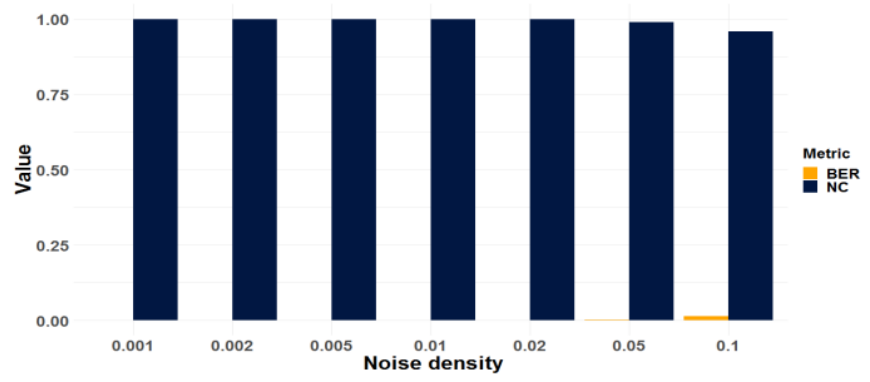

Figure 13. Lena image robustness results against Gaussian noise different densities 


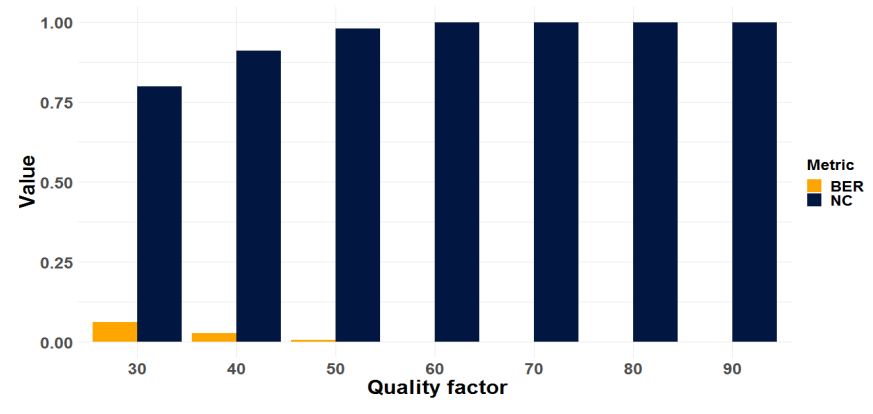

Figure 14. Lena image robustness results against JPEG compression various quality factors

\subsection{Quantitative comparison}

In reason of affirming the proposed scheme's effectiveness, comparison with other methods in terms of $\mathrm{NC}, \mathrm{BER}$, and processing time was done in this section.

\subsubsection{Robustness comparison}

Table 5 expose NC and BER results of the proposed work compared to other techniques suggested by Singh [32], Kazemivash and Moghaddam [33], Zear et al. [34], Kalra et al. [35] and Ariatmanto and Ernawan [36], the cover image used for comparison is Lena as common host image with other techniques in comparison, and the watermark is the plus sign logo. it can be remarked that the proposed algorithm shows maximum robustness against Slat \& Pepper noise with $1 \%$ density, sharing this result with the method [35], for $\mathrm{NC}$ values obtained from different Gaussian noise densities are 0.61 and 0.95 for $1 \%$ density for the schemes [32, 35], 0.91 for $0.5 \%$ density for the method [33] and 0.99 for $0.1 \%$ density for the technique [36], however, the method in hands offer higher robustness than previously reported techniques. This method revealed the highest $\mathrm{NC}$ results for various rotation angles $\left(5^{\circ}\right.$ and $90^{\circ}$ ) compared to other techniques. The proposed technique demonstrated the top results for the rest methods, in addition, the proposed method depicted low BER values for all the attack types, which enhances the potency of the suggested algorithm.

\subsubsection{Operational time}

The bar chart depicted in Figure 15, illustrates the embedding and extraction execution time of the proposed framework and other methods, it is measured in seconds. Overall, it can be seen that the proposed scheme scored fast total processing between imperceptibility and the robustness. The superior results achieved by the proposed algorithm confirm its effectiveness against divers' attacks in comparison with different techniques, furthermore, the rapid execution time, where the embedding process took $0.2224 \mathrm{~s}$, against $0.1046 \mathrm{~s}$ for the retrieving process, Table 6 shows the computational cost of several techniques including the proposed scheme. From the illustrated results we can extrapolate that the proposed scheme is the most rapid, and basing in this fact, the proposed technique is appropriate to be used in real life.

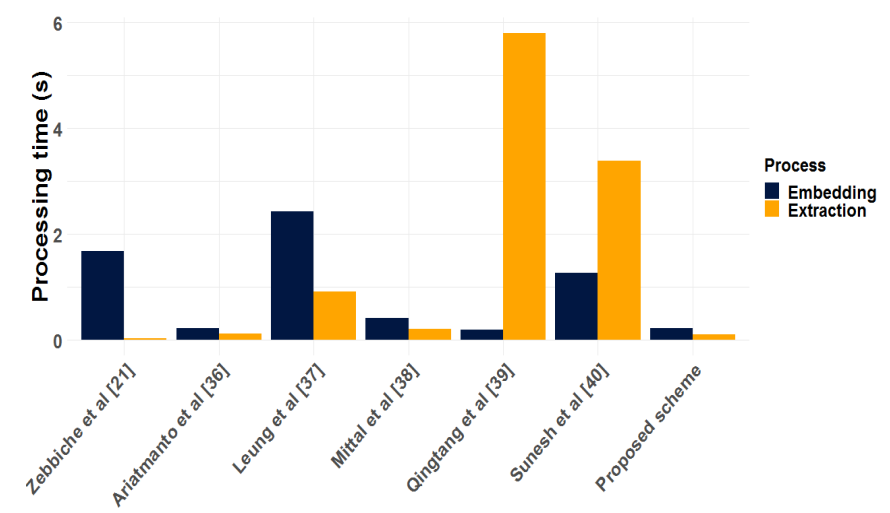

Figure 15. Embedding and extraction processes operational time comparison

Table 4. Extracted watermark results under several attack types

\begin{tabular}{|c|c|c|c|c|}
\hline Attacks & Image & $\mathbf{N C}$ & BER & Extracted Watermark \\
\hline \multirow{3}{*}{$\&$ pepper $($ density $=1 \%)$} & Lena & 1 & 0 & \\
\hline & Hand & 1 & 0 & \\
\hline & Brain & 1 & 0 & \\
\hline & Lena & 0.962 & 0.0117 & \\
\hline & Hand & 0.940 & 0.0195 & \\
\hline$\&$ pepper $($ density $=5 \%)$ & Brain & 0.965 & 0.0107 & \\
\hline \multirow{3}{*}{ Gaussian noise $($ mean $=0 ;$ density $=1 \%)$} & Lena & 0.996 & 0.0010 & \\
\hline & Hand & 0.983 & 0.0039 & \\
\hline & Brain & 0.993 & 0.0020 & \\
\hline \multirow[b]{3}{*}{ Gaussian noise $($ mean $=0 ;$ density $=2 \%$ ) } & Lena & 0.946 & 0.0166 & \\
\hline & Hand & 0.948 & 0.0156 & \\
\hline & Brain & 0.940 & 0.0186 & \\
\hline \multirow{3}{*}{ Speckle noise $($ density $=1 \%$ ) } & Lena & 1 & 0 & \\
\hline & Hand & 1 & 0 & \\
\hline & Brain & 1 & 0 & \\
\hline \multirow[b]{3}{*}{ Speckle noise $($ variance $=5 \%)$} & Lena & 0.990 & 0.0029 & \\
\hline & Hand & 0.987 & 0.0039 & \\
\hline & Brain & 1 & 0 & \\
\hline
\end{tabular}




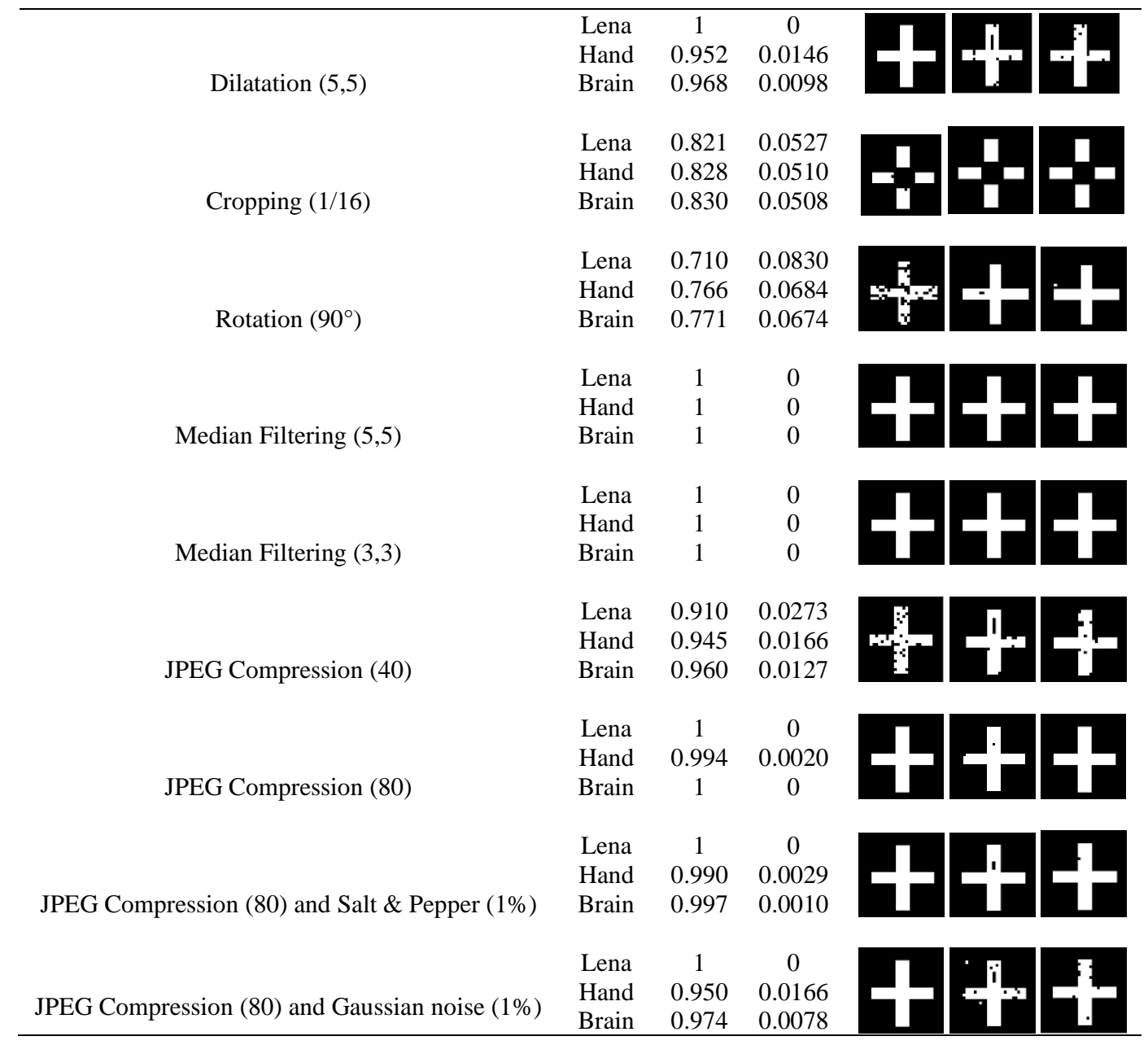

Table 5. Robustness comparison with other methods in terms of NC and BER

\begin{tabular}{|c|c|c|c|c|c|c|c|c|c|c|c|c|}
\hline \multirow[t]{2}{*}{ Attacks } & \multicolumn{2}{|c|}{ Singh [32] } & \multicolumn{2}{|c|}{$\begin{array}{c}\text { Kazemivash et a } \\
{[33]}\end{array}$} & \multicolumn{2}{|c|}{$\begin{array}{c}\text { Zear et al. } \\
{[34]}\end{array}$} & \multicolumn{2}{|c|}{$\begin{array}{c}\text { Kalra et al. } \\
{[35]}\end{array}$} & \multicolumn{2}{|c|}{$\begin{array}{c}\text { Ariatmanto et al } \\
{[36]}\end{array}$} & \multicolumn{2}{|c|}{$\begin{array}{c}\text { Proposed } \\
\text { method }\end{array}$} \\
\hline & $\mathrm{NC}$ & BER & $\mathrm{NC}$ & BER & NC & BER & $\mathrm{NC}$ & BER & $\mathrm{NC}$ & BER & $\mathrm{NC}$ & BER \\
\hline $\begin{array}{c}\text { Salt \& Pepper noise } \\
(1 \%)\end{array}$ & 0.80 & 0 & 0.96 & 0.0156 & 0.7569 & 0 & 1 & 0 & 0.93 & 0.0684 & 1 & 0 \\
\hline Gaussian noise $(1 \%)$ & 0.61 & 0.0400 & NA & NA & NA & NA & 0.95 & 0.081 & NA & NA & 0.99 & 0.001 \\
\hline $\begin{array}{l}\text { Gaussian noise } \\
\qquad(0.5 \%)\end{array}$ & NA & NA & 0.91 & 0.0322 & NA & NA & NA & NA & NA & NA & 1 & 0 \\
\hline $\begin{array}{c}\text { Gaussian noise } \\
(0.1 \%)\end{array}$ & NA & NA & NA & NA & NA & NA & NA & NA & 0.99 & 0.0098 & 1 & 0 \\
\hline Speckle noise (1\%) & 0.88 & 0 & NA & NA & 0.9024 & 0.1119 & 1 & 0 & NA & NA & 1 & 0 \\
\hline $\begin{array}{l}\text { Speckle noise } \\
(0.5 \%)\end{array}$ & NA & NA & 0.99 & 0.0010 & 0.9860 & 0 & NA & NA & NA & NA & 1 & 0 \\
\hline Rotation $\left(5^{\circ}\right)$ & 0.03 & 0.4970 & NA & NA & NA & NA & NA & NA & 0.49 & 0.5098 & 0.53 & 0.1250 \\
\hline Rotation $\left(90^{\circ}\right)$ & NA & NA & 0.10 & 0.5205 & NA & NA & NA & NA & NA & NA & 0.71 & 0.0830 \\
\hline Median filter $(3 * 3)$ & NA & NA & 0.90 & 0.0391 & 0.0025 & 0 & 0.77 & 0.3590 & 0.99 & 0.0020 & 1 & 0 \\
\hline JPEG (60) & 0.95 & 0 & NA & NA & 0.9679 & 0 & 0.79 & 0.3350 & 1 & 0 & 1 & 0 \\
\hline JPEG (80) & 0.98 & 0 & 1 & 0 & 0.9812 & 0 & 1 & 0 & 1 & 0 & 1 & 0 \\
\hline $\begin{array}{l}\text { JPEG }(80) \text { and Salt } \\
\& \text { Pepper noise }(1 \%)\end{array}$ & 0.80 & 0.0057 & NA & NA & NA & NA & NA & NA & NA & NA & 1 & 0 \\
\hline $\begin{array}{c}\text { JPEG }(80) \text { and Salt } \\
\& \text { Pepper noise } \\
(0.2 \%)\end{array}$ & NA & NA & NA & NA & 0.9074 & 0 & NA & NA & NA & NA & 1 & 0 \\
\hline $\begin{array}{c}\text { JPEG (80) and } \\
\text { Gaussian noise (1\%) }\end{array}$ & 0.89 & 0 & NA & NA & NA & NA & NA & NA & 0.99 & 0.0020 & 0.99 & 0.0020 \\
\hline $\begin{array}{l}\text { JPEG }(80) \text { and } \\
\text { Gaussian noise } \\
(0.2 \%)\end{array}$ & NA & NA & NA & NA & 0.8135 & 0 & NA & NA & NA & NA & 1 & 0 \\
\hline $\begin{array}{l}\text { Salt \& Pepper noise } \\
(0.3 \%) \text { and Median } \\
\text { filter }(3,3)\end{array}$ & NA & NA & NA & NA & NA & NA & NA & NA & 0.99 & 0.0020 & 1 & 0 \\
\hline
\end{tabular}


Table 6. Computational cost for different schemes

\begin{tabular}{ccc}
\hline Methods & Embedding & Extraction \\
\hline Proposed method & 0.2224 & 0.1046 \\
Zebbiche et al. [21] & 1.6766 & 0.0269 \\
Ariatmanto et al. [36] & 0.2188 & 0.1250 \\
Leung et al. [37] & 2.43 & 0.92 \\
Mittal et al. [38] & 0.4071 & 0.2076 \\
Su and Chen [39] & 0.1948 & 5.8023 \\
Malik and Rama Kishore et al. [40] & 1.26 & 3.38 \\
\hline
\end{tabular}

\section{CONCLUSIONS}

In this research, a blind watermarking algorithm for medical images DTCWT and NSCT based was investigated. The method mechanism uses binary logos as watermarks and color medical images as hosts, the suggested technique performance depends on the quantization step which is the main link opens the doors for the proposed method to be implemented in several applications. To conclude, the proposed algorithm advantages are highlighted in the next points:

- High robustness against divers' signal processing manipulations'

- Strong performance versus JPEG compression

- Excellent visual quality

- Very fast processing time.

As future perspective, the watermark will be encrypted using a solid cryptosystem, which will lead to optimum efficiency.

\section{ACKNOWLEDGMENT}

This work was supported by Directorate General for Scientific Research and Technological Development (DGRSDT).

\section{REFERENCES}

[1] Bhardwaj, R., Sharma, V. (2016). Image steganography based on complemented message and inverted bit LSB substitution. Procedia Computer Science, 93: 832-838. https://doi.org/10.1016/j.procs.2016.07.245

[2] Vasudev, R. (2016). A review paper on digital watermarking techniques \& its applications. International Journal of Science and Research (IJSR), $5(6)$ : https://doi.org/10.21275/v5i6.nov164699

[3] Singh, R., Shaw, D., Alam, M. (2015). Experimental studies of LSB watermarking with different noise. Procedia Computer Science, 54: 612-620. https://doi.org/10.1016/j.procs.2015.06.071

[4] Kuraparthi, S., Kollati, M., Kora, P. (2019). Robust optimized discrete wavelet transform-singular value decomposition based video watermarking. Traitement du Signal, 36(6):

$565-573$ https://doi.org/10.18280/ts.360612

[5] Najafi, E., Loukhaoukha, K. (2019). Hybrid secure and robust image watermarking scheme based on SVD and sharp frequency localized contourlet transform. Journal of Information Security and Applications, 44: 144-156. https://doi.org/10.1016/j.jisa.2018.12.002

[6] Chen, L., Zhao, J. (2017). Contourlet-based image and video watermarking robust to geometric attacks and compressions. Multimedia Tools and Applications, 77(6) 7187-7204. https://doi.org/10.1007/s1 1042-017-4628-7

[7] Das, S., Banerjee, M., Chaudhuri, A. (2017). An improved DCT based image watermarking robust against JPEG compression and other attacks. International Journal of Image, Graphics and Signal Processing, 9(9): 40-50. https://doi.org/10.5815/ijigsp.2017.09.05

[8] Li, Y., Yao, S., Yang, K., Tan, Y., Zhang, Q. (2019). A high-imperceptibility and histogram-shifting data hiding scheme for JPEG images. IEEE Access, 7: 73573-73582. https://doi.org/10.1109/ACCESS.2019.2920178

[9] Bamatraf, A., Ibrahim, R., Salleh, M. (2010). Digital watermarking algorithm using LSB. 2010 International Conference on Computer Applications and Industrial Electronics, Kuala Lumpur, pp. 155-159. https://doi.org/10.1109/iccaie.2010.5735066

[10] Qin, C., Ji, P., Zhang, X., Dong, J., Wang, J. (2017). Fragile image watermarking with pixel-wise recovery based on overlapping embedding strategy. Signal Processing, 138: 280-293. https://doi.org/10.1016/j.sigpro.2017.03.033

[11] Hamidi, M., El Haziti, M., Cherifi, H., Hassouni, M. (2018). Hybrid blind robust image watermarking technique based on DFT-DCT and Arnold transform. Multimedia Tools and Applications, 77(20): 2718127214. https://doi.org/10.1007/s11042-018-5913-9

[12] Ansari, I., Pant, M. (2017). Multipurpose image watermarking in the domain of DWT based on SVD and ABC. Pattern Recognition Letters, 94: 228-236. https://doi.org/10.1016/j.patrec.2016.12.010

[13] Yuan, Z., Su, Q., Liu, D., Zhang, X. (2020). A blind image watermarking scheme combining spatial domain and frequency domain. The Visual Computer. https://doi.org/10.1007/s00371-020-01945-y

[14] Ernawan, F., Kabir, M.N. (2018). A robust image watermarking technique with an optimal DCTpsychovisual threshold. IEEE Access, 6: 20464-20480. https://doi.org/10.1109/ACCESS.2018.2819424

[15] Poonam, Arora, S. (2018). A DWT-SVD based robust digital watermarking for digital images. Procedia Computer $\quad$ Science, 132: 1441-1448. https://doi.org/10.1016/j.procs.2018.05.076

[16] Islam, M., Roy, A., Laskar, R. (2018). SVM-based robust image watermarking technique in LWT domain using different sub-bands. Neural Computing and Applications, 32(5): 1379-1403. https://doi.org/10.1007/s00521-0183647-2

[17] Li, D., Deng, L., Bhooshan Gupta, B., Wang, H., Choi, C. (2019). A novel CNN based security guaranteed image watermarking generation scenario for smart city applications. Information Sciences, 479: 432-447. https://doi.org/10.1016/j.ins.2018.02.060

[18] Abraham, J., Paul, V. (2019). An imperceptible spatial domain color image watermarking scheme. Journal of King Saud University - Computer and Information Sciences, $\quad 31(1)$ : 125-133. https://doi.org/10.1016/j.jksuci.2016.12.004

[19] Kingsbury, N.G. (1998). The dual-tree complex wavelet transform: A new technique for shift invariance and directional filters. In: IEEE Digital Signal Processing Workshop, Bryce Canyon.

[20] Yadav, J., Sehra, K. (2018). Large scale dual tree complex wavelet transform based robust features in PCA 
and SVD subspace for digital image watermarking. Procedia Computer Science, 132: 863-872. https://doi.org/10.1016/j.procs.2018.05.098

[21] Zebbiche, K., Khelifi, F., Loukhaoukha, K. (2018). Robust additive watermarking in the DTCWT domain based on perceptual masking. Multimedia Tools and Applications, $\quad$ 77(16): 21281-21304. https://doi.org/10.1007/s11042-017-5451-x

[22] Liu, J., Li, J., Ma, J., Sadiq, N., Bhatti, U., Ai, Y. (2019). A robust multi-watermarking algorithm for medical images based on DTCWT-DCT and Henon map. Applied Sciences, 9(4): 700. https://doi.org/10.3390/app9040700

[23] Alkhathami, M., Han, F., Van Schyndel, R. (2013). Fingerprint image watermarking approach using DTCWT without corrupting minutiae. 2013 6th International Congress on Image and Signal Processing (CISP), Hangzhou, pp. 1717-1723. https://doi.org/10.1109/CISP.2013.6743953

[24] Wang, W., Ning, Y., Hu, Y., Yang, Y. (2019). Research on a digital watermarking algorithm for color image based on dual-tree complex wavelet transform (DTCWT). 2019 2nd World Conference on Mechanical Engineering and Intelligent Manufacturing (WCMEIM), Shanghai, China, pp. 443-446. https://doi.org/10.1109/WCMEIM48965.2019.00093

[25] Kumar, C.A., Rajendra Naik, B. (2019). A new encrypted image watermarking based on DTCWT and random pixel exchange. 2019 5th International Conference on Advanced Computing \& Communication Systems (ICACCS), Coimbatore, India, pp. 567-570. https://doi.org/10.1109/ICACCS.2019.8728398

[26] Do, M., Vetterli, M. (2005). The contourlet transform: An efficient directional multiresolution image representation. IEEE Transactions on Image Processing, 14(12):

2091-2106 https://doi.org/10.1109/tip.2005.859376

[27] Ghaz, A., Kpalma, K., Bounoua, A. (2014). NSCT edge enhancement for SIFT key points extraction. IOSR Journal of VLSI and Signal Processing, 4(2): 84-90. https://doi.org/10.9790/4200-04218490

[28] Da Cunha, A.L., Zhou, J., Do, M. (2006). The nonsubsampled contourlet transform: Theory, design, and applications. IEEE Transactions on Image Processing, 15(10): 3089-3101. https://doi.org/10.1109/tip.2006.877507

[29] Nam, S., Mun, S., Ahn, W., Kim, D., Yu, I., Kim, W., Lee, H. (2020). NSCT-based robust and perceptual watermarking for DIBR 3D images. IEEE Access, 8: 93760-93781.

https://doi.org/10.1109/access.2020.2994966

[30] Wang, X., Zhang, S., Wen, T., Yang, H., Niu, P. (2019).
Coefficient difference-based watermark detector in nonsubsampled contourlet transform domain. Information Sciences, 503: 274-290. https://doi.org/10.1016/j.ins.2019.06.059

[31] "MedPix", Medpix.nlm.nih.gov, 2020. [Online]. Available: https://medpix.nlm.nih.gov/home, accessed on 26- Oct- 2020.

[32] Singh, A.K. (2019). Robust and distortion control dual watermarking in LWT domain using DCT and error correction code for color medical image. Multimedia Tools and Applications, 78(21): 30523-30533. https://doi.org/10.1007/s11042-018-7115-X

[33] Kazemivash, B., Moghaddam, M. (2016). A robust digital image watermarking technique using lifting wavelet transform and firefly algorithm. Multimedia Tools and Applications, 76(20): 20499-20524. https://doi.org/10.1007/s11042-016-3962-5

[34] Zear, A., Singh, A., Kumar, P. (2016). A proposed secure multiple watermarking technique based on DWT, DCT and SVD for application in medicine. Multimedia Tools and Applications, 77(4): 4863-4882. https://doi.org/10.1007/s11042-016-3862-8

[35] Kalra, G.S., Talwar, R., Sadawarti, H. (2013). Digital image watermarking in frequency domain using ECC and dual encryption technique. Research Journal of Applied Sciences, Engineering and Technology, 6(18): 3365-3371. https://doi.org/10.19026/rjaset.6.3649

[36] Ariatmanto, D., Ernawan, F. (2020). An improved robust image watermarking by using different embedding strengths. Multimedia Tools and Applications, 79(17-18): 12041-12067. https://doi.org/10.1007/s11042-01908338-x

[37] Leung, H., Cheng, L., Liu, F. (2012). Robust digital image watermarking scheme using wave atoms with multiple description coding. EURASIP Journal on Advances in Signal Processing, 2012(1): 245. https://doi.org/10.1186/1687-6180-2012-245

[38] Mittal, M., Kaushik, R., Verma, A., Kaur, I., Goyal, L.M. Roy, S., Kim, T. (2020). Image watermarking in curvelet domain Using edge surface blocks. Symmetry, 12(5): 822. https://doi.org/10.3390/sym12050822

[39] Su, Q., Chen, B. (2017). Robust color image watermarking technique in the spatial domain. Soft Computing, 22(1): 91-106. https://doi.org/10.1007/s00500-017-2489-7

[40] Malik, S., Rama Kishore, R. (2020). Spiking neural network based scrambled watermark hiding in lowfrequency region of digital image. Journal of Information and Optimization Sciences, 41(2): 437-459. https://doi.org/10.1080/02522667.2020.1723939 